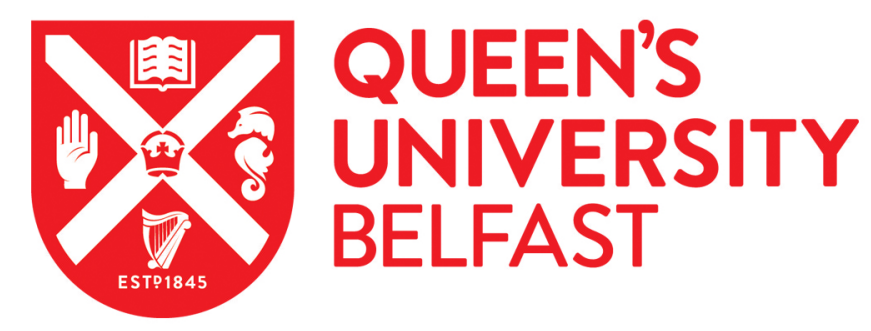

\title{
Efficient excitation of dielectric-loaded surface plasmon-polariton waveguide modes at telecommunication wavelengths
}

Holmgaard, T., Bozhevolnyi, S. I., Markey, L., Dereux, A., Krasavin, A. V., Bolger, P., \& Zayats, A. V. (2008). Efficient excitation of dielectric-loaded surface plasmon-polariton waveguide modes at telecommunication wavelengths. Physical Review B (Condensed Matter), 78(16), [165431]. https://doi.org/10.1103/PhysRevB.78.165431

Published in:

Physical Review B (Condensed Matter)

Document Version:

Publisher's PDF, also known as Version of record

Queen's University Belfast - Research Portal:

Link to publication record in Queen's University Belfast Research Portal

Publisher rights

@2008 The American Physical Society

\section{General rights}

Copyright for the publications made accessible via the Queen's University Belfast Research Portal is retained by the author(s) and / or other copyright owners and it is a condition of accessing these publications that users recognise and abide by the legal requirements associated with these rights.

Take down policy

The Research Portal is Queen's institutional repository that provides access to Queen's research output. Every effort has been made to ensure that content in the Research Portal does not infringe any person's rights, or applicable UK laws. If you discover content in the Research Portal that you believe breaches copyright or violates any law, please contact openaccess@qub.ac.uk. 


\title{
Efficient excitation of dielectric-loaded surface plasmon-polariton waveguide modes at telecommunication wavelengths
}

\author{
Tobias Holmgaard* \\ Department of Physics and Nanotechnology, Aalborg University, Skjernvej 4A, DK-9220 Aalborg Øst, Denmark \\ Sergey I. Bozhevolnyi \\ Department of Physics and Nanotechnology, Aalborg University, Skjernvej 4A, DK-9220 Aalborg Øst, Denmark \\ and Institute of Sensors, Signals, and Electrotechnics (SENSE), University of Southern Denmark, \\ Niels Bohrs Allé 1, DK-5230 Odense M, Denmark \\ Laurent Markey and Alain Dereux \\ Institut Carnot de Bourgogne, UMR 5209 CNRS-Université de Bourgogne, 9 Av. A. Savary, BP 47 870, \\ F-21078 DIJON Cedex, France
}

\begin{abstract}
Alexey V. Krasavin, Pádraig Bolger, and Anatoly V. Zayats
Centre for Nanostructured Media, IRCEP, The Queens University of Belfast, Belfast BT7 INN, United Kingdom

(Received 5 May 2008; revised manuscript received 10 September 2008; published 31 October 2008)

The excitation of surface plasmon-polariton (SPP) waveguide modes in subwavelength dielectric ridges deposited on a thin gold film has been characterized and optimized at telecommunication wavelengths. The experimental data on the electromagnetic mode structure obtained using scanning near-field optical microscopy have been directly compared to full vectorial three-dimensional finite element method simulations. Two excitation geometries have been investigated where SPPs are excited outside or inside the dielectric tapered region adjoint to the waveguide. The dependence of the efficiency of the SPP guided mode excitation on the taper opening angle has been measured and modeled. Single-mode guiding and strong lateral mode confinement of dielectric-loaded SPP waveguide modes have been characterized with the near-field measurements and compared to the effective-index method model.
\end{abstract}

DOI: 10.1103/PhysRevB.78.165431

PACS number(s): 73.20.Mf, 71.36.+c, 68.37.Uv, 42.79.Gn

\section{INTRODUCTION}

The recent acceleration of the research on photonic components based on surface plasmon polaritons (SPPs) is motivated by the expectation that plasmonics is able to combine the asset of optical components, with respect to bandwidth, and the asset of electronic components, with respect to size. ${ }^{1,2}$ SPPs are collective oscillations in the surface plasma of a metal coupled to an optical wave, bound to, and propagating along, the metal-dielectric interface. ${ }^{3,4}$ In addition to the signal carrying properties, SPPs have been found useful for other applications, such as achieving active photonic functionalities $^{5}$ and enhanced sensing and detection of biomolecules, ${ }^{6}$ all utilizing the strong SPP field confinement near the metal-dielectric interface. The strong lateral confinement in the direction perpendicular to the SPP propagation is essential for the development of compact plasmonic circuits since it ensures smaller bend losses and higher density of components. It should be noted, however, that strong confinement is often achieved by reducing the SPP field in the dielectric, therefore, with an increase in the propagation loss. Thus, the choice of optimum waveguide structure often is subject to a trade-off. In addition to a demand of strong lateral confinement and low propagation loss, single-mode guiding by the plasmonic waveguide structures is usually desired in order to avoid various mode dispersion and interference effects.

Several metal-dielectric structures have been investigated for guiding SPPs. Channel SPP waveguides, where lateral confinement is achieved by fabricating a square or $\mathrm{V}$ shaped groove in a flat metal surface, have been subject to extensive theoretical and experimental investigations in recent years. ${ }^{7-11}$ Rectangular metal stripes, where lateral confinement is achieved by shrinking the lateral extension of a thin metal film, have been investigated theoretically ${ }^{12-14}$ and characterized experimentally. ${ }^{15-17}$ Lateral SPP confinement has also been achieved by utilizing nanostructured periodic variations in the metal surface surrounding the waveguide ${ }^{18}$ or by using chains of closely spaced metal nanoparticles as waveguides. ${ }^{19,20}$

An alternative and technologically simple approach to achieving SPP waveguiding with strong lateral confinement is the use of dielectric stripes deposited on a thin metal film, thereby achieving dielectric-loaded surface plasmonpolariton (DLSPP) waveguides (DLSPPWs). Such waveguides rely on the same principle used in conventional integrated optics, where high-index-contrast waveguides (waveguides with a core refractive index much higher than that of the surrounding cladding) are used to achieve a small mode size. ${ }^{21}$ Dielectric optical elements for focusing, reflection and refraction, ${ }^{22}$ and DLSPPWs (Refs. 23-26) have been analyzed, fabricated, and characterized at near-infrared wavelengths. The mode confinement, propagation loss, and single-mode conditions of DLSPPWs have been analyzed theoretically by the effective-index method (EIM) and finite element method (FEM) at telecommunication wavelengths, ${ }^{27}$ as have basic passive plasmonic components such as bends, 
splitters, and directional couplers. ${ }^{28}$ The excitation and propagation of DLSPPW modes in straight waveguides and bends have also recently been reported at telecommunication wavelengths ${ }^{29}$ giving prospect of the realization of compact plasmonic components based on DLSPPWs. In this paper different schemes for exciting DLSPPW modes are analyzed and the mode confinement and propagation loss are characterized at telecommunication wavelengths.

The paper is organized as follows. In Sec. II the experimental and theoretical techniques employed in the characterization of DLSPPWs are presented and a description of the investigated sample is given. In Sec. III the results of the FEM calculations, the EIM calculations, and scanning nearfield optical microscope (SNOM) characterization are presented. Finally in Sec. IV a discussion of the obtained results is given and we offer our conclusions.

\section{METHODS AND MATERIALS}

In the characterization of the excitation and propagation of DLSPPW modes a SNOM imaging system is utilized and in modeling the FEM and the EIM are applied. After a description of the sample configuration, a brief introduction to the methods is presented.

\section{A. Sample configuration}

The investigated sample consists of polymethylmethacrylate (PMMA) ridges $\left(n_{r}=1.493\right)$ lying on an $\sim 50 \mathrm{~nm}$ thin gold film thermally evaporated on a $170 \mu \mathrm{m}$ thick glass substrate. The fabrication process is briefly described as follows: after the initial substrate cleaning and gold deposition, a PMMA film was spin coated from $950 \mathrm{~K}$ molecular weight PMMA resist (Allresist Gmbh, Ref. AR-P 671.04) and soft baked. Photolithography was performed on a Süss Microtech MJB4 mask aligner, equipped with UV250 optics, using the vacuum mode, and the resist was finally developed in methyl-isobutyl-ketone. Due to in coupling considerations the DLSPPWs are extended by funnel tapering structures [Fig. 1(a)].

Four different in coupling funnels with a fixed width of $10 \mu \mathrm{m}$ and a length varying from 10 to $25 \mu \mathrm{m}$ in steps of $5 \mu \mathrm{m}$ are realized in a DLSPPW block. The optimum waveguide dimensions, i.e., ridge height and width, were deduced by simultaneously considering mode confinement and propagation loss while retaining the demand of single-mode DLSPPWs. ${ }^{27}$ Investigation of the sample with a scanning electron microscope (SEM) reveals a waveguide width of $w \sim 500 \mathrm{~nm}$ [Fig. 1(b)]. Application of an atomic force microscope (AFM) in the investigation of the sample yields a waveguide height of $h \sim 550 \mathrm{~nm}$ [Figs. 1(c) and 1(d)]. These waveguide dimensions are found in accordance with the calculated design parameters presented in Ref. 27.

\section{B. SNOM measurements}

The experimental setup for near-field characterization of DLSPPW structures consists of an aperture SNOM operating in collection mode [Fig. 2]. The sample under investigation is placed on a glass prism using index matching immersion

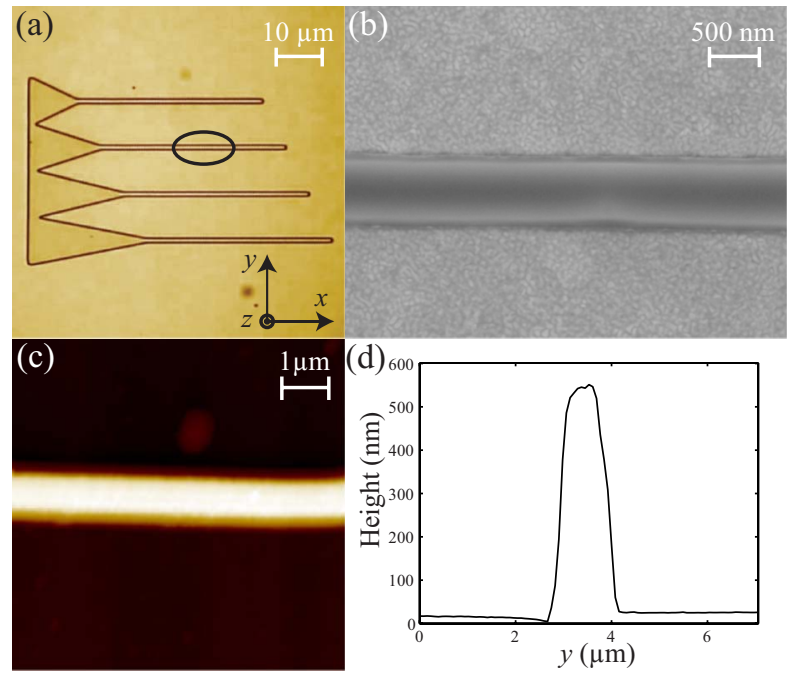

FIG. 1. (Color online) (a) Microscope image of a DLSPPW block with four different tapering structures. (b) SEM image of a straight section of a waveguide, revealing a waveguide width of $w \sim 500 \mathrm{~nm}$. (c) AFM image of a straight section of a waveguide and (d) cross sectional profile of the AFM image revealing a waveguide height of $h \sim 550 \mathrm{~nm}$.

oil. SPPs are excited utilizing two different schemes, but common for both is that a lensed fiber is used to focus a $p$-polarized Gaussian beam to a spot size of $\sim 15 \mu \mathrm{m}$ at the gold surface opposite to the DLSPPW components, thus exciting SPPs by means of the Kretschmann-Raether configuration. ${ }^{4}$ An uncoated etched fiber tip is raster scanned across the sample surface, whereby topographic and nearfield optical images are achieved. In one excitation scheme SPPs are excited at the gold-air interface $\sim 20 \mu \mathrm{m}$ before the in coupling funnel by matching the lateral component of the incident wave vector to that of SPPs propagating along, and bound to, the gold-air interface (see Fig. 3). In this configuration the funnels have the effect of coupling the SPPs into DLSPPW modes by total internal reflection (TIR) in the dielectric funnel. Moreover, close to the waveguides these funnels will screen the propagation of SPPs at the gold-air interface. In the other excitation scheme DLSPPs are excited directly in the funnel region by matching the lateral compo-

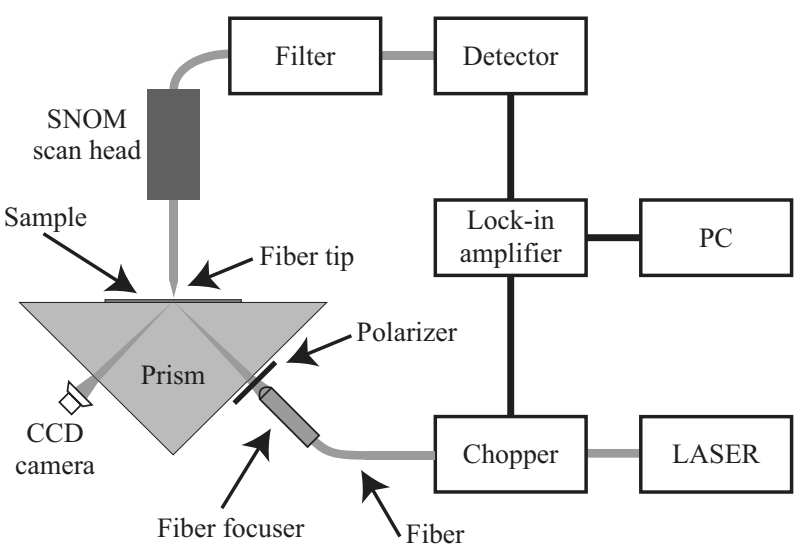

FIG. 2. The experimental setup of the SNOM imaging system used to obtain near-field optical images of the DLSPPW structures. 


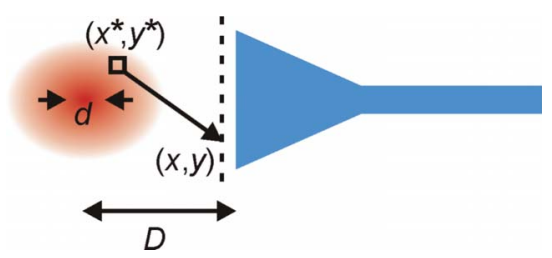

FIG. 3. (Color online) Schematic drawing illustrating the calculation of the incident SPP field distribution used in the 3D FEM calculations.

nent of the incident wave vector to that of SPPs propagating along, and bound to, the gold-PMMA interface. This is done by utilizing a high-index prism $(n=1.73)$.

\section{FEM calculation}

Numerical simulation of funnel efficiency is performed using full three-dimensional (3D) FEM calculations, proved to be very rigorous in simulation of various photonic and plasmonic structures. ${ }^{27,30,31}$ This method solves Maxwell's equations in frequency domain without any simplifications. As a refractive index for gold a tabulated value $n_{\mathrm{Au}}$ $=0.55-11.5 i$, taken from Ref. 33, was used. Simulation of the whole setup including attenuated total reflection (ATR) coupling of light into a SPP wave propagating at the metal surface before the funnel proved too demanding in three dimensions in terms of the simulation domain volume. To overcome this problem, only the essential processes of coupling into the DLSPPW mode using a funnel and its further propagation are simulated while calculating the SPP wave fields incident on the funnel analytically. The incident SPP field distribution at a distance of $1 \mu \mathrm{m}$ before the funnel was calculated using a standard method of calculating diffracted fields in optics. ${ }^{32}$ The laser spot, of Gaussian profile and with diameter $d=10 \mu \mathrm{m}$, incident at an angle of $\psi=40^{\circ}$ was placed at a distance $D=15 \mu \mathrm{m}$ from the funnel (see Fig. 3).

The phase distribution of the excited SPP wave was derived from the condition of resonant SPP excitation $k_{x}$ $=\operatorname{Re}\left(k_{\mathrm{SPP}}\right)$. Thus the following expression for the $y$ component of the magnetic field, completely defining the incident SPP field, appears:

$$
\begin{aligned}
H_{y}(x, y) \propto & \int A_{0}\left(x^{*}, y^{*}\right) \exp \left[i \phi_{0}\left(x^{*}\right)\right] K_{\mathrm{damp}}\left(x, y, x^{*}, y^{*}\right) \\
& \times \exp \left[i \phi\left(x, y, x^{*}, y^{*}\right)\right] \cos (\alpha) d x^{*} d y^{*},
\end{aligned}
$$

where

$$
A_{0}\left(x^{*}, y^{*}\right)=\exp \left[-x^{* 2} /\left(d /(2 \cos \psi)^{2}\right)-y^{* 2} /(d / 2)^{2}\right]
$$

represents the amplitude distribution of the incident beam,

$$
\phi_{0}\left(x^{*}\right)=\operatorname{Re}\left(k_{\mathrm{SPP}}\right) x^{*},
$$

its phase distribution,

$$
K_{\mathrm{damp}}\left(x, y, x^{*}, y^{*}\right)=\frac{\exp \left(-\sqrt{\left(x-x^{*}\right)^{2}+\left(y-y^{*}\right)^{2}} \operatorname{Im}\left(k_{\mathrm{SPP}}\right)\right)}{\sqrt[4]{\left(x-x^{*}\right)^{2}+\left(y-y^{*}\right)^{2}}},
$$

represents damping of SPP wave due to the radial spreading and the absorption damping, and

$$
\cos (\alpha)=\left(x-x^{*}\right) / \sqrt{\left(x-x^{*}\right)^{2}+\left(y-y^{*}\right)^{2}}
$$

represents the cosine of the SPP incident angle.

\section{EIM calculation}

The EIM is one of the standard methods for mode analysis of optical and SPP waveguides, attractive due to its simplicity and low demand for computational capabilities. The method is known to yield reasonably accurate results for DLSPPW modes far from cutoff, ${ }^{27}$ which is the case for the DLSPPW modes considered in this work. In the EIM the two-dimensional cross section of the DLSPPW is considered and solved for guided modes by consecutively considering two one-dimensional waveguide structures. In the first step of the EIM the waveguide geometry is considered to have infinite lateral extend, thus the problem is reduced to finding bound modes in a multilayer waveguide. In this step the waveguide is considered as an air-PMMA-gold-glass structure, where the air and glass layers are considered semiinfinite in extend, and the mode $p$-polarized. In the second step a three layer structure with infinite vertical extend and $s$-polarized fields is considered. The mode effective index found in the first step is used to represent the middle layer (ridge region), enclosed by two semi-infinite layers with mode index set to that of a SPP wave at a gold-air interface. By solving this multilayer structure the mode indexes for bound SPP modes supported by the DLSPPW geometry are found. ${ }^{27}$

\section{RESULTS}

The DLSPPW block with four different tapering designs has been characterized by using near-field imaging with a SNOM in order to determine the efficiency for coupling in SPPs excited at the bare gold-air interface, i.e., the fraction of the power of the incident SPP field which is coupled into a guided DLSPPW mode [Figs. 4(a) and 4(b)]. The same in coupling structures have also been investigated using the FEM, where the incident SPP field distribution has been calculated as described in Sec. II C [Figs. 4(c) and 4(d)].

Both experimental and theoretical results show an increase in the coupling efficiency with increasing funnel length. Furthermore the characterization shows that the longer tapers, which have smaller funnel angle, cause less scattering at the end of the tapering region as the incident SPP wave hits the dielectric-air boundary under a larger angle. Thus it experiences TIR not only at the first intersection with the funnel boundary, as is the case with the shortest funnel. In both experimental and simulation field maps, oscillations in the field intensity can be observed along both taper and waveguide. By analyzing the modes existing in the funnel (particularly their effective indexes, defining the inter- 

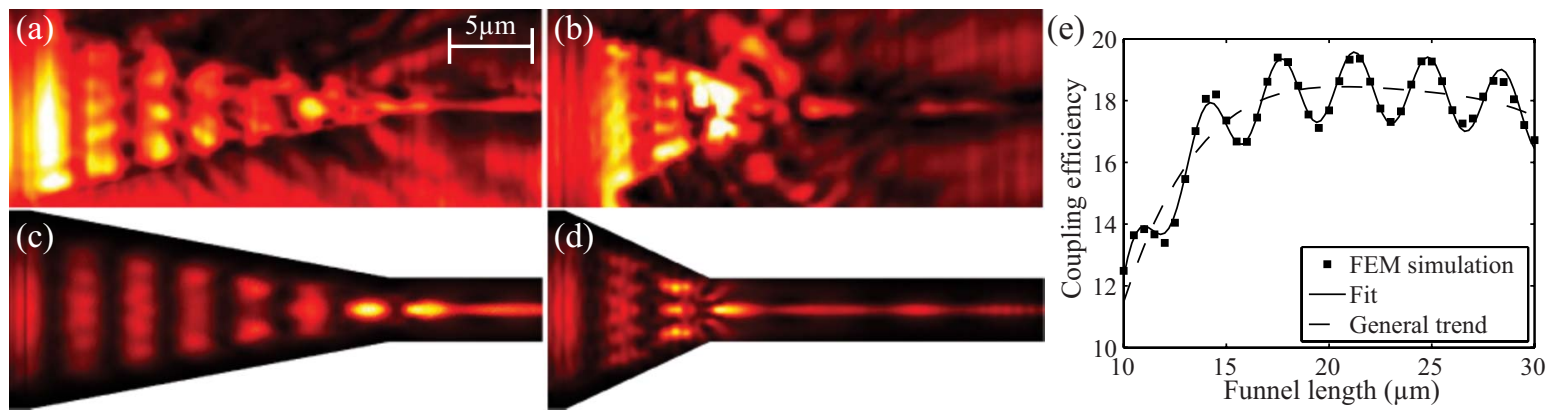

FIG. 4. (Color online) Near-field optical images of the tapering region of the DLSPPWs at the free space excitation wavelength $\lambda$ $=1550 \mathrm{~nm}$. [(a) and (c)] SNOM and FEM images, respectively, of a $10 \times 25 \mu \mathrm{m}^{2}$ funnel. [(b) and (d)] SNOM and FEM images, respectively, of a $10 \times 10 \mu \mathrm{m}^{2}$ funnel. (e) Coupling efficiency as a function of taper length, calculated using the FEM, where the incident SPP field is kept identical for all funnel lengths. The reconstructed SPP power flow at the beginning of the straight waveguide section is normalized to the power flow incident on the funnel, thus obtaining the coupling efficiency. The images in (a)-(d) share the scale shown in (a).

ference period) it was found that the oscillations in the FEM and SNOM images can be explained by the interference between the fundamental $\mathrm{TM}_{00}$ mode, excited when the SPP hits the taper, and freely propagating waves, part of which also propagate inside the taper before spreading out in the process of diffraction. A detailed analysis of the coupling efficiency performed with the FEM reveals that the efficiency initially increases with funnel length until reaching a level where the increase in efficiency, due to better focusing, is balanced by the increased loss caused by longer propagation through the funnel [Fig. 4(e)]. For the shortest taper the losses are found to be caused by SPPs escaping through the sides of the funnel, accounting for $\sim 50 \%$ loss, SPP scattering through the top of the funnel, accounting for $\sim 15 \%$, and absorption losses from propagation through the funnel, accounting for $\sim 10 \%$, while a smaller fraction is lost due to reflection from the initial funnel boundary and excitation of freely propagating waves in the ridge waveguide. For longer funnels the fraction lost due to propagation increases and the losses due to scattering through the sides of the funnel and excitation of freely propagating waves in the ridge decreases, whereas the other loss factors are more or less constant. It is logical that with further taper length increase the propagation loss becomes dominant and the performance of the taper will be decreasing. Thus the optimal value of the taper length is estimated to be $\sim 20 \mu \mathrm{m}$.

In the determination of the coupling efficiency great care has been taken to eliminate influence of the oscillations along the waveguide, which can be observed in Figs. 4(c) and 4(d). By analyzing the interference pattern along the waveguide it was possible to derive only the desired SPP signal, which was then used to evaluate the coupling efficiency. The oscillations in the coupling efficiency as a function of funnel length [Fig. 4(e)] are found to be primarily caused by the propagating waves in the funnel, which are diffracted at the end of the taper and thus couple to the SPP mode in the waveguide. This contribution will interfere with the main one (originating from the $\mathrm{TM}_{00}$ mode in the funnel) and produce the oscillations observed in the coupling efficiency. Depending with which phase difference these SPP and propagating waves in the taper arrive at the taper end, a different result of interference of the SPP waveguide modes produced from each of them occurs. Moreover, the periodic- ity of the phase difference of the taper SPP and light modes are given by their interference in the taper $\sim 3.5 \mu \mathrm{m}$. The same periodicity is observed in the coupling efficiency, which confirms this explanation.

The method of excitation of SPPs at the gold-air interface and subsequent coupling into bound DLSPPW modes is attractive due to its simplicity; however, it has some apparent disadvantages, which become clear when considering profiles of the near-field optical images (Fig. 5). An averaged cross-sectional profile of the near-field optical image taken at the straight waveguide section just after the tapering region [see Fig. 5(c)] reveals that a strongly confined DLSPPW mode indeed is excited; however, it is also apparent that scattered light from the funnel along with copropagating SPPs at the gold-air interface (not screened by the funnel) result in a large degree of background. This is undesired and a potential problem when realizing plasmonic components such as bends and splitters, where the waveguide is displaced, as the copropagating fields will scatter off the waveguides. ${ }^{29}$ A profile of the near-field optical image taken parallel to the waveguide, through the funnel and the waveguide, shows that the DLSPP field is damped when propagating through the funnel as the effect of propagation loss is larger than the focusing effect of the funnel [Fig. 5(d)]. The oscillations that can be observed are caused by interference between the $\mathrm{TM}_{00}$ mode and propagating modes as discussed above.

The alternative excitation scheme, where DLSPP modes are excited directly in the tapering structure, is attractive as it is expected that no copropagating SPP fields at the gold-air interface are excited due to the much higher effective index of the dielectric funnel region. This is indeed observed by SNOM characterization of a $10 \times 25 \mu \mathrm{m}^{2}$ funnel (Fig. 6).

An averaged cross-sectional profile of the near-field optical image taken at the straight waveguide section just after the end of the funnel shows a highly confined DLSPPW mode with no copropagating SPPs at the gold-air interface and almost no apparent scattering originating from the end of the funnel [Fig. 6(c)]. A profile of the near-field optical signal taken parallel to the waveguide shows a strong buildup of the DLSPP mode in the tapering region [Fig. 6(d)]. This effect is strongly opposed to that observed in the other excitation method [Fig. 5(d)] and is caused by several factors all con- 

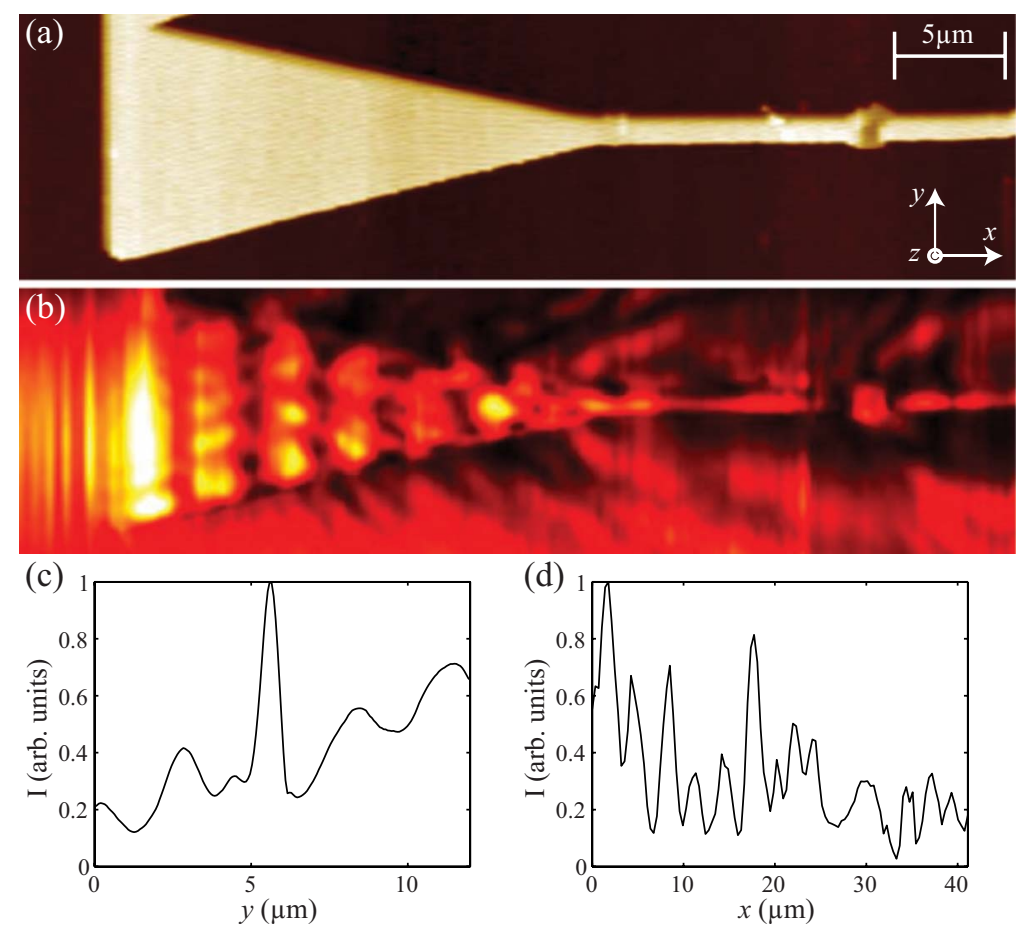

FIG. 5. (Color online) SNOM images of a $10 \times 25 \mu \mathrm{m}^{2}$ funnel in coupling, where SPPs are excited at the gold-air interface outside the tapering region (in the area to the left of the funnel, not shown in the images), for the free space excitation wavelength $\lambda=1550 \mathrm{~nm}$. [(a) and (b)] Topographical and near-field optical images, respectively, obtained with the SNOM imaging system. (c) Averaged cross sectional profile of the near-field optical image taken at the straight waveguide section just after the end of the funnel. (d) Averaged profile of the near-field optical image taken parallel with the waveguide, through the funnel and waveguide regions. tributing to a stronger optical signal toward the end of the tapering region. First the excitation of SPPs by the attenuated TIR method causes a strong initial increase in SPP intensity, as the propagation loss initially has a small effect. Second the tapering structure has a focusing effect toward the funnel end due to TIR of DLSPPs, which will also result in a stronger optical signal toward the funnel end. Finally the imaging process further enhances this trend as near-field optical modes with lower effective indexes are picked up more strongly with the tapered fiber, and as the mode effective index of a dielectric ridge decreases with width this also contributes to a stronger optical signal toward the funnel end. Due to the strong excitation of DLSPPWs without causing copropagating SPPs and scattering of SPPs, this excitation scheme is found highly attractive and superior to the previously described excitation method and has thus been used in the achievement of the results presented in the rest of the paper.

The propagation and confinement of DLSPPW modes in straight waveguides have been characterized by considering a waveguide section $\sim 50 \mu \mathrm{m}$ after the tapering region using SNOM imaging at the free space excitation wavelength $\lambda$
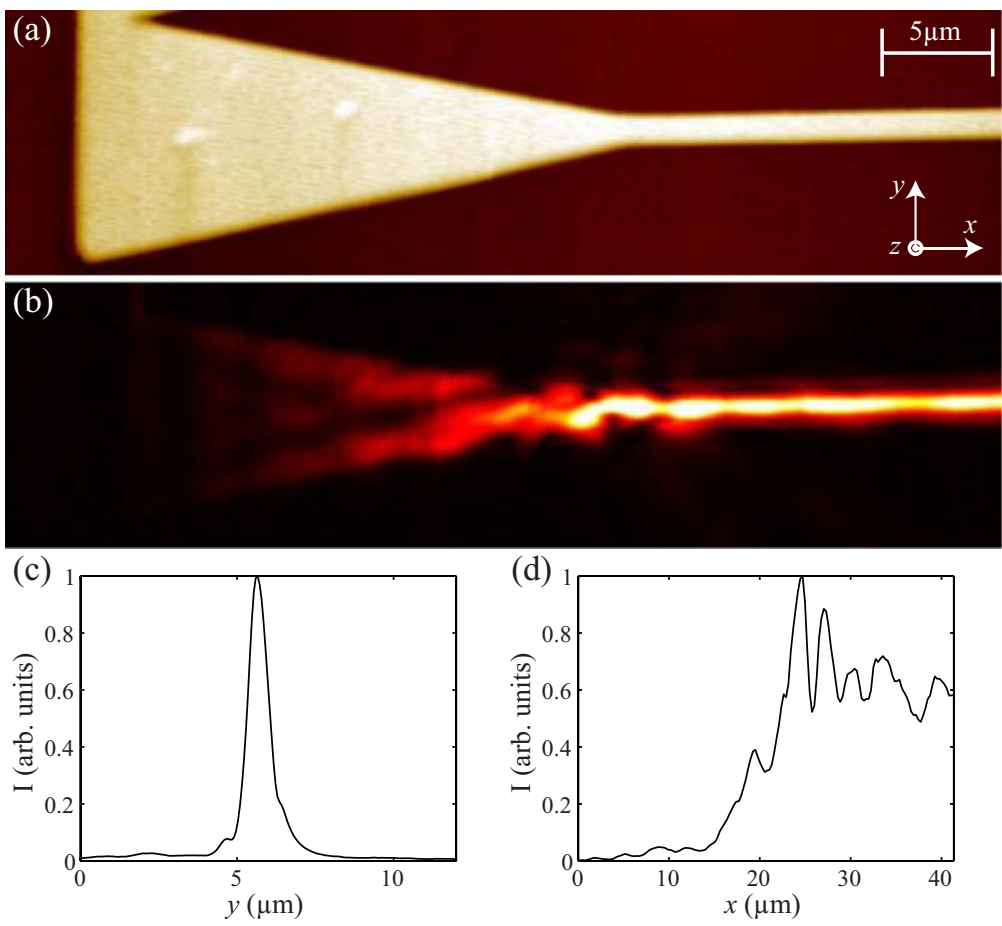

FIG. 6. (Color online) SNOM images of a $10 \times 25 \mu \mathrm{m}^{2}$ funnel in coupling, where SPPs are excited at the gold-PMMA interface inside the tapering region, for the free space excitation wavelength $\lambda=1550 \mathrm{~nm}$. [(a) and (b)] Topographical and near-field optical images, respectively, obtained with the SNOM. (c) Averaged cross-sectional profile of the near-field optical image taken at the straight waveguide section just after the end of the funnel. (d) Averaged profile of the near-field optical image taken parallel with the waveguide through the funnel and waveguide regions. 

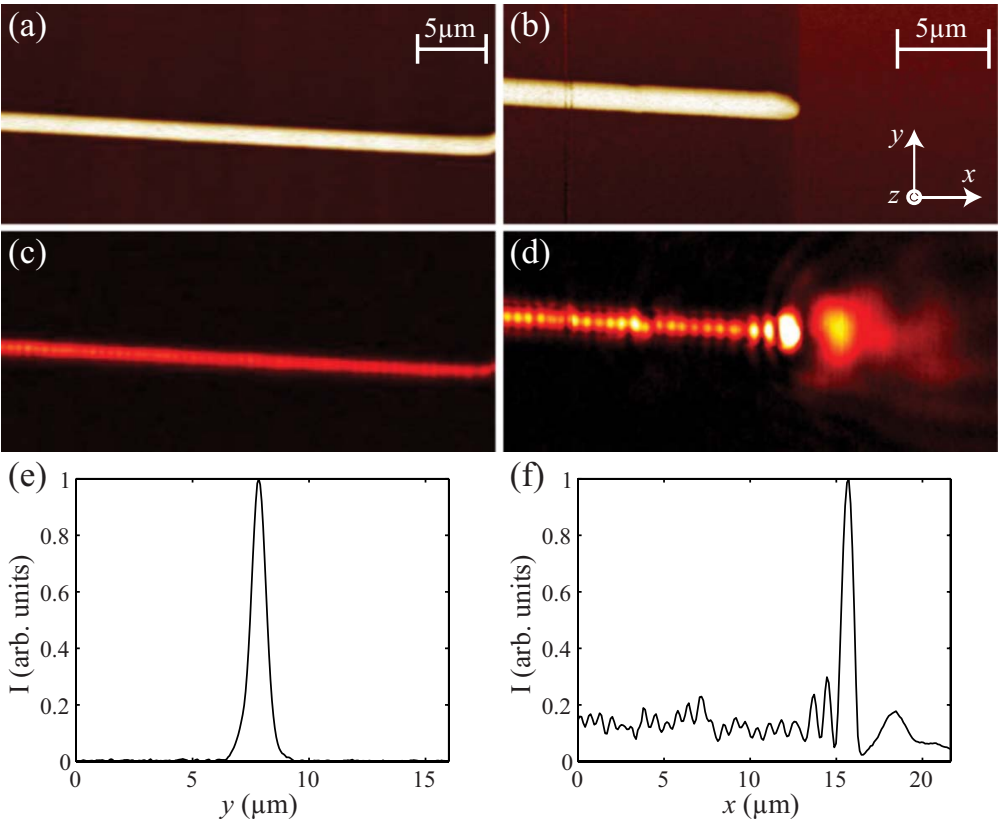

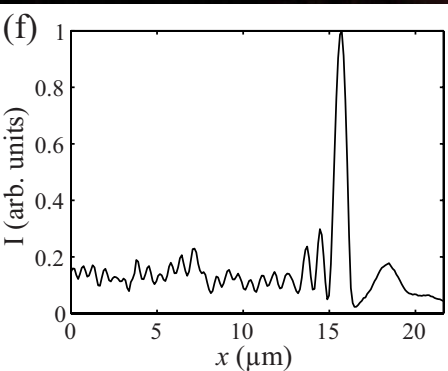

FIG. 7. (Color online) SNOM images of a straight DLSPPW section and a waveguide termination, recorded at the free space excitation wavelength $\lambda=1550 \mathrm{~nm}$. [(a) and (c)] Topographical and near-field optical images, respectively, of a straight DLSPPW section. [(b) and (d)] Topographical and near-field optical images, respectively, of a waveguide termination. (e) Cross sectional profile of the near-field optical image in (c) yielding a FWHM of $\sim 743$ nm. (f) Profile of the near-field optical image in (d) taken parallel with the waveguide, revealing an interference pattern with a period of $\Lambda \sim 700 \mathrm{~nm}$.
$=1550 \mathrm{~nm}$ [Figs. 7(a) and 7(c)]. The near-field optical image shows a strongly confined DLSPPW mode, and the absence of mode beating confirms that the designed DLSPPW structure indeed only supports a single TM mode. The mode width is investigated by making an averaged cross-sectional profile of the near-field optical signal, revealing a full width at half maximum (FWHM) of $\sim 743 \mathrm{~nm}$ [Fig. 7(e)], indicating subwavelength confinement. The waveguide termination region has also been investigated by using SNOM imaging, and it is found that even after propagation over more than $\sim 100 \mu \mathrm{m}$ a strong DLSPPW signal exists as can be observed from the strong scattering at the waveguide termination [Figs. 7(b) and 7(d)]. An interference pattern can be observed in the near-field optical signal, and by considering a profile taken along the waveguide the interference period is found to be $\Lambda \sim 700 \mathrm{~nm}$ [Fig. 7(f)]. This interference pattern is thus found to be caused by interference between the forward propagating DLSPPW $\left(\mathrm{TM}_{00}\right)$ mode with mode effective index $N_{\text {eff }} \sim 1.21$ (EIM calculations at $\lambda=1550 \mathrm{~nm}$ yields $N_{\text {eff }}=1.22$ ) and a backward propagating light wave with effective index close to 1 . The origin of the backward propagating light wave is the waveguide termination, where the DLSPPW mode is scattered from. No significant back reflection of the DLSPPW mode inside the ridge for the termination is expected due to the, in this respect, relatively small difference in mode effective index between the DLSPPW mode and a SPP at the gold-air interface and the fact that the termination of the dielectric ridge is not completely abrupt. This is supported from FEM simulations of a DLSPPW termination, where the reflectivity has been estimated to be $\sim 5 \%$.

The propagation loss in the fabricated DLSPPWs has been investigated by using near-field imaging with a SNOM to characterize a straight waveguide section at different wavelengths in the telecommunication range (Fig. 8). From EIM calculations the DLSPPWs are expected to show similar properties at the investigated telecommunication wavelengths, however, with an increase in propagation length
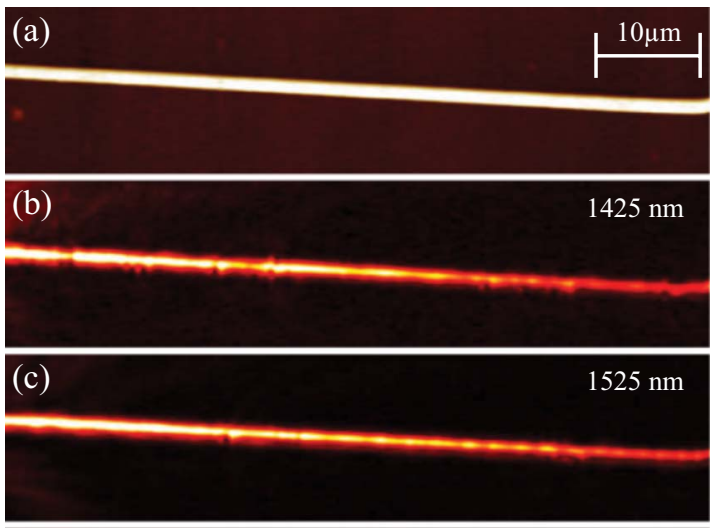

(d)

$1625 \mathrm{~nm}$

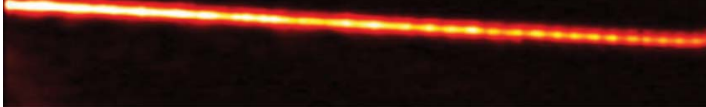

FIG. 8. (Color online) SNOM images of a straight waveguide at different free space excitation wavelengths. (a) Topographic image, (b) near-field optical image for $\lambda=1425 \mathrm{~nm}$, where the propagation length is determined to $L$ $=46 \mu \mathrm{m}$, (c) near-field optical image for $\lambda=1525 \mathrm{~nm}$, where $L$ $=52 \mu \mathrm{m}$, and (d) near-field optical image for $\lambda=1625 \mathrm{~nm}$, where $L=65 \mu \mathrm{m}$. [(e) and (f)] Mode effective index and propagation length, respectively, as a function of wavelength, calculated by utilizing the EIM (fully drawn line) and measured with the SNOM (squares) (Ref. 33). 
with increasing wavelength, at the expense of poorer mode confinement (lower mode effective index) [Figs. 8(e) and $8(\mathrm{f})$. This trend is confirmed from contemplation of the obtained near-field optical images, where an exponential fit to an averaged profile taken along the waveguide yields propagation lengths of $L=46 \mu \mathrm{m}, L=52 \mu \mathrm{m}$, and $L=65 \mu \mathrm{m}$ at the free space excitation wavelengths $\lambda=1425 \mathrm{~nm}, \lambda$ $=1525 \mathrm{~nm}$, and $\lambda=1625 \mathrm{~nm}$, respectively [Figs. 8(b)-8(d)]. It is observed that the measured propagation lengths in all cases are longer than those expected from the EIM calculations conducted for the DLSPPWs having the designed ridge width and height. This can be caused by several effects, where the most likely are found to be discrepancy in waveguide dimensions, e.g., smaller waveguide width than designed or a slightly trapezoidal shape of the waveguide, which can result in quite large deviations in propagation length. ${ }^{27}$ Another possible cause could be discrepancy between the actual gold refractive index and that used in the EIM calculations.

\section{DISCUSSION AND CONCLUSIONS}

The excitation and propagation of DLSPPW modes have been characterized at telecommunication wavelengths by utilizing calculations made with the FEM and the EIM and by near-field measurements with a SNOM. The performance of different funnel shaped tapering structures for coupling into DLSPPW modes, utilizing the Kretschmann-Raether configuration, has been investigated, and it was found that funnels with smaller opening angle, i.e., larger length to width ratio, proved better for in coupling. By analysis of field intensity maps obtained by numerical simulations with FEM and by near-field optical measurements with a SNOM it is found that the larger incident angle to the dielectric funnel boundary results in less scattering and better focusing toward the funnel end, thus resulting in stronger coupling into bound DLSPPW modes. This results in an increasing coupling efficiency with funnel length, until a certain length of $\sim 20 \mu \mathrm{m}$, where the propagation loss through the funnel becomes dominant, thereby decreasing the efficiency. Two different excitation schemes, one where SPPs are excited at the bare gold-air interface and coupled into DLSPPW modes using the funnel and other where DLSPPs are excited directly in the dielectric funnel by using a high-index prism, were char- acterized by using near-field imaging with a SNOM. The second method is found superior, as it avoids excitation of copropagating SPPs at the gold-air interface, and as it minimizes scattering of SPPs off the funnel.

A straight waveguide section has been characterized with a SNOM imaging system in order to evaluate the mode confinement and propagation loss. The obtained images confirmed the expectation of single-mode propagation, as no mode beatings could be observed. A cross sectional profile of a near-field optical image obtained for $\lambda=1550 \mathrm{~nm}$ showed a strong lateral confinement with a FWHM of $\sim 743 \mathrm{~nm}$. The mode effective index of the bound DLSPPW mode has been evaluated by contemplation of the waveguide termination where the forward propagating DLSPPW mode interferes with a backward propagating light wave originating from scattering at the waveguide termination. The mode effective index has been evaluated to be $N_{\text {eff }} \sim 1.21$ for $\lambda$ $=1550 \mathrm{~nm}$, in very good agreement with the EIM calculated index of $N_{\text {eff }}=1.22$. The propagation lengths, measured at the telecommunication wavelengths $\lambda=1425 \mathrm{~nm}, \lambda=1525 \mathrm{~nm}$, and $\lambda=1625 \mathrm{~nm}$ by making an exponential fit to near-field optical images obtained with a SNOM, showed an increase with wavelength expected from theoretical investigations. The measured propagation lengths are longer than those expected from the EIM calculations, which is found to be a result of slightly differing waveguide dimensions and possible variations in the gold refractive indexes from those used in EIM calculations.

In conclusion we have demonstrated efficient excitation of DLSPPW modes with low background noise, single-mode propagation, strong confinement, and reasonably low propagation loss, all issues which are crucial for realizing efficient plasmonic components. We conduct further investigations in this area.

\section{ACKNOWLEDGMENTS}

The authors thank Thomas Søndergaard for help in developing a multilayer waveguide program used in this work and Jens Rafaelsen for assistance in SEM imaging of the samples. The financial support of the EC FP6 STREP PLASMOCOM (Grant No. IST 034754) is acknowledged. A.V.K., P.B., and A.V.Z. acknowledge the financial support of EPSRC-GB (U.K.).

\footnotetext{
*holmgaard@nano.aau.dk

${ }^{1}$ W. L. Barnes, A. Dereux, and T. W. Ebbesen, Nature (London) 424, 824 (2003).

${ }^{2}$ E. Ozbay, Science 311, 189 (2006).

${ }^{3}$ V. M. Agranovich and D. L. Mills, Surface PolaritonsElectromagnetic Waves at Surfaces and Interfaces, 1st ed. (North-Holland, Amsterdam, 1982).

${ }^{4}$ H. Raether, Surface Plasmons—on Smooth and Rough Surfaces and on Gratings, 1st ed. (Springer-Verlag, Berlin, 1988).

${ }^{5}$ A. V. Zayats, I. I. Smolyaninov, and A. Maradudin, Phys. Rep. 408, 131 (2005).
}

${ }^{6}$ S. Lal, S. Link, and N. J. Halas, Nat. Photonics 1, 641 (2007).

${ }^{7}$ I. V. Novikov and A. A. Maradudin, Phys. Rev. B 66, 035403 (2002).

${ }^{8}$ D. K. Gramotnev and D. F. P. Pile, Appl. Phys. Lett. 85, 6323 (2004).

${ }^{9}$ S. I. Bozhevolnyi, Opt. Express 14, 9467 (2006).

${ }^{10}$ S. I. Bozhevolnyi, V. S. Volkov, E. Devaux, J.-Y. Laluet, and T. W. Ebbesen, Nature (London) 440, 508 (2006).

${ }^{11}$ S. I. Bozhevolnyi and J. Jung, Opt. Express 16, 2676 (2008).

${ }^{12}$ P. Berini, Phys. Rev. B 61, 10484 (2000).

${ }^{13}$ R. Zia, M. D. Selker, and M. L. Brongersma, Phys. Rev. B 71, 
165431 (2005).

${ }^{14}$ J. Jung, T. Søndergaard, and S. I. Bozhevolnyi, Phys. Rev. B 76, 035434 (2007).

${ }^{15}$ T. Nikolajsen, K. Leosson, I. Salakhutdinov, and S. I. Bozhevolnyi, Appl. Phys. Lett. 82, 668 (2003).

${ }^{16}$ K. Leosson, T. Nikolajsen, A. Boltasseva, and S. I. Bozhevolnyi, Opt. Express 14, 314 (2006).

${ }^{17}$ P. Berini, R. Charbonneau, and N. Lahoud, Nano Lett. 7, 1376 (2007).

${ }^{18}$ S. I. Bozhevolnyi, J. Erland, K. Leosson, P. M. W. Skovgaard, and J. M. Hvam, Phys. Rev. Lett. 86, 3008 (2001).

${ }^{19}$ M. Quinten, A. Leitner, J. R. Krenn, and F. R. Aussenegg, Opt. Lett. 23, 1331 (1998).

${ }^{20}$ S. A. Maier, P. G. Kik, H. A. Atwater, S. Meltzer, E. Harel, B. E. Koel, and A. A. G. Requicha, Nature Mater. 2, 229 (2003).

${ }^{21}$ C. Manolatou, S. G. Johnson, S. Fan, P. R. Villeneuve, H. A. Haus, and J. D. Joannopoulos, J. Lightwave Technol. 17, 1682 (1999).

${ }^{22}$ A. Hohenau, J. R. Krenn, A. L. Stepanov, A. Drezet, H. Ditlbacher, B. Steinberger, A. Leitner, and F. R. Aussenegg, Opt. Lett. 30, 893 (2005)

${ }^{23}$ C. Reinhardt, S. Passinger, B. N. Chichkov, C. Marquart, I. P. Radko, and S. I. Bozhevolnyi, Opt. Lett. 31, 1307 (2006).
${ }^{24}$ B. Steinberger, A. Hohenau, H. Ditlbacher, A. L. Stepanov, A. Drezet, F. R. Aussenegg, A. Leitner, and J. R. Krenn, Appl. Phys. Lett. 88, 094104 (2006).

${ }^{25}$ B. Steinberger, A. Hohenau, H. Ditlbacher, F. R. Aussenegg, A. Leitner, and J. R. Krenn, Appl. Phys. Lett. 91, 081111 (2007).

${ }^{26}$ S. Massenot, J. Grandidier, A. Bouhelier, G. C. des Francs, L. Markey, J.-C. Weeber, A. Dereux, J. Renger, M. U. Gonzàlez, and R. Quidant, Appl. Phys. Lett. 91, 243102 (2007).

${ }^{27}$ T. Holmgaard and S. I. Bozhevolnyi, Phys. Rev. B 75, 245405 (2007).

${ }^{28}$ A. V. Krasavin and A. V. Zayats, Appl. Phys. Lett. 90, 211101 (2007).

${ }^{29}$ T. Holmgaard, S. I. Bozhevolnyi, L. Markey, and A. Dereux, Appl. Phys. Lett. 92, 011124 (2008).

${ }^{30}$ M. P. Nezhad, K. Tetz, and Y. Fainman, Opt. Express 12, 4072 (2004).

${ }^{31}$ A. V. Krasavin, A. S. Schwanecke, and N. I. Zheludev, J. Opt. A, Pure Appl. Opt. 8, S98 (2006).

${ }^{32}$ E. Hecht, Optics, 4th ed. (Addison-Wesley, San Francisco, 2002).

${ }^{33}$ E. D. Palik, Handbook of Optical Constants of Solids, 1st ed. (Academic, New York, 1985). 